\title{
Potencialidad y viabilidades de los procesos de evaluación por pares abierto: el caso de Encrucijadas. Revista Crítica de Ciencias Sociales
}

\author{
Dara Medina Chirino ${ }^{1}$; Juan Manuel García González²; Rafael Grande³; David Prieto \\ Serrano $^{4}$
}

Recibido: 3 de abril de 2017 / Aceptado: 9 de junio de 2017

\begin{abstract}
Resumen. Los procesos de evaluación de las revistas académicas y científicas se han transformado en los últimos años debido al incremento tanto del número de revistas como del volumen de artículos recibidos. Además, el uso de internet ha generado un fuerte aperturismo de la ciencia, con cada vez más revistas en acceso abierto. Los equipos editoriales han tenido que adaptarse y decidirse por un modelo de evaluación que se adecue a este nuevo entorno. Dado este contexto, en este artículo se expone el caso particular del sistema de evaluación de Encrucijadas. Revista Crítica de Ciencias Sociales. Esta revista plantea un modelo de evaluación abierta en el que se conozcan tanto autores como evaluadores durante todo el proceso de revisión. Mediante un cuestionario precodificado semiabierto, se analiza cuál fue la percepción sobre ese sistema de evaluación tanto de autores como evaluadores. La conclusión principal es que hay una aceptación del modelo por parte de los dos colectivos, si bien hay ligeras diferencias por edad, sexo y colectivo
\end{abstract}

Palabras clave: revistas, proceso editorial, evaluación por pares abierta, evaluación por doble ciego.

\section{[en] Potentiality and feasibility of open peer review: the case of Encrucija- das. Revista Crítica de Ciencias Sociales}

\begin{abstract}
Evaluation processes of scientific and academic journals have been transformed in recent years due to the increase in both the number of journals and the volume of papers received. Furthermore, the use of Internet has generated a strong openness of science, with more and more open access journals. Editorial teams have had to adapt to this new setting and decide on an evaluation model. In this context, this paper presents the case of the evaluation system of the Spanish journal Encrucijadas. Revista Crítica de Ciencias Sociales. This journal proposes an open peer review in which both authors and reviewers are known each other during all the evaluation process. We use a precoded semi-
\end{abstract}

1 Socióloga, profesional independiente

E-mail: dara.mchirino@gmail.com

2 Universidad Pablo de Olavide

Email: jmgargon@gmail.com

3 Universidad de Málaga

Email: rgrande@uma.es

4 Universidad Nacional de Educación a Distancia

Email: davidprietoserrano@hotmail.com

Las y los autores firman en nombre del Comité Editorial de Encrucijadas. Revista Crítica de Ciencias Sociales 
open questionnaire in order to analyze the opinion of both authors and reviewers about this type of evaluation. We conclude that there is an acceptance of the model of review by the two groups, although there are some slight differences by age, sex, and type of contribution.

Keywords: journals, editorial process, open peer review, double blind peer review.

Sumario. 1. Introducción. 2. Un estudio de caso: la evaluación abierta de Encrucijadas. 3. Datos y métodos. 4. Resultados. 5. Conclusiones. 6. Referencias bibliográficas

Cómo citar: Chirino, D., García González, J.M., Grande, R., Prieto Serrano, D. (2017) Potencialidad y viabilidades de los procesos de evaluación por pares abierto: el caso de Encrucijadas. Revista Crítica de Ciencias Sociales, en Teknokultura 14, 35-56.

\section{Introducción}

En la actualidad cualquier investigador es consciente de que los procesos de evaluación por pares son el eje sobre el que se articulan los sistemas científicos, más aún ante el predominio del formato paper como formato de difusión y de los índices de impacto como acreditación de la actividad investigadora. Sin embargo, la evaluación por pares como método de arbitraje científico es un sistema consolidado recientemente, que sólo logra su hegemonía en los procesos de publicación y asignación de recursos tras la II Guerra Mundial, y que ha sido problemático desde sus orígenes(Csiszar, 2016). El debate actual sobre esta práctica (funcionesdisfunciones, calidad, sesgos, desigualdades, ralentización, sobrecargas, etc.) hace necesario reflexionar sobre los pros y los contras de los diferentes modelos de evaluación, centrándonos en las diferencias entre el modelo abierto y el modelo del doble ciego.

En este contexto, el presente artículo tiene como objetivo estudiar, desde una perspectiva de auto-reflexión, las actitudes y valoraciones que autores y evaluadores hacen del proceso de evaluación por pares abierto de la revista Encrucijadas, durante el proceso editorial comprendido entre los años 2011 y 2013, en los que se publicaron seis volúmenes monográficos ${ }^{5}$, en comparación con el sistema clásico de doble ciego.

Encrucijadas. Revista Crítica de Ciencias Sociales es una publicación semestral independiente que lanzó su primer número en junio de 2011. En su finalidad inicial pretendía ser un punto de encuentro, por un lado, entre distintas disciplinas dentro de las ciencias sociales abordando diversas temáticas y, por otro lado, entre autores de diversas procedencias (universidad, centros de investigación, mundo laboral, independientes, etc.).

Desde que se creó la revista hasta el día de hoy, sin desatender esos objetivos, se han ido enfrentando diferentes retos al calor de las nuevas imposiciones del sistema científico, de las tendencias de publicación académicas y de la evolución propia de la revista; tratando de no perder la perspectiva crítica, tanto en el material

5 Vol.1 (2011): Trabajo, sindicalismo y crisis económica, Vol.2 (2011): Espacios urbanos: perspectivas y conflictos, Vol.3 (2012): Estado y soberanía en Europa, Vol.4 (2012): Metodologías en las Ciencias Sociales: disputas y consensos, Vol.5 (2013): Mujeres, géneros, feminismos, Vol.6 (2013): Mundo rural: población, territorio, poder. 
publicado como en el proceso editorial, lo que se traduce en no dejar de cuestionar el proceso de selección y evaluación de artículos, así como el papel que debe desempeñar el Comité Editorial en este proceso.

El debate sobre con qué sistema de evaluación por pares externos trabajar ha estado presente desde el momento inicial del proyecto, entendiéndolo como un aspecto fundamental a la hora de alcanzar los objetivos de rigor y calidad científicas y, más allá de esto, tratando de buscar la comodidad de evaluadores y autores frente a un entorno que, por lo general, dibuja cada vez de forma más hostil esa triple relación autor-editor-evaluador. Por ello, desde su creación Encrucijadas decide valorar de forma experimental alternativas al sistema de doble ciego de revisión por pares.

Encrucijadas parte de la idea que la evaluación no deja de ser un proceso complejo que no está libre de los sesgos ni de los vicios que condicionan todo trabajo analítico y de revisión, lo que no hace fácil pronunciarse con rotundidad por un tipo de modelo u otro. No obstante, desde el primer momento sus editores apuestan por un modelo de evaluación abierta (open peer review) apoyándose en dos aspectos. Primero, este sistema de evaluación por pares era más cuidadoso y reportaba menos fragilidad que el modelo de doble ciego imperante en las revistas españolas. Frente a una posible lasitud de las evaluaciones de doble ciego donde el evaluador opina y dictamina respaldado en el anonimato, el modelo abierto exige más trabajo y más cuidado al evaluador, lo cual hace, por lo general, los dictámenes más ambiguos, pero, por otro lado, rompe el tipo de relación clásica autor-evaluador. Segundo, optar por esta vía suponía, en cierta medida, un experimento para poner en cuestión los sistemas hegemónicos de evaluación por pares externos. Es decir, queríamos probar si un sistema abierto podía garantizar evaluaciones y publicaciones rigurosas. En suma, la propia organización del proceso editorial -en especial el modelo de evaluación- también buscaba la perspectiva crítica que persigue Encrucijadas.

Pese a la notable expansión durante los últimos años de las revistas académicas que han adoptado el acceso abierto (Open Access)(Prado Arreaza, Velasco Ortuño, González Padial, \& Sanromán Rodríguez, s. f.), el arbitraje abierto de los trabajos es aún minoritario. Aun así, desde que en 1997 la revista The Medical Journal of Australia lanzase la primera prueba a este modelo, el open peer review ha ido creciendo paulatinamente en el campo de las revistas científicas, principalmente en publicaciones de las ciencias llamadas "duras" del ámbito anglosajón. Sin embargo, no ha sido así en el campo de las ciencias sociales, y aún menos en el ámbito de habla hispana. Una revisión a las especificaciones de las principales revistas de ciencias sociales según el índice de impacto elaborado por In-Recs o el índice $\mathrm{H}$ apuntaría a la de Encrucijadas como una experiencia inédita en su contexto, dado que la gran mayoría opta por el sistema de doble ciego, si bien es cierto que otras muchas no especifican su sistema de evaluación.

Si bien, no existe una definición normalizada ni unos rasgos delimitado de la evaluación abierta, Ford (2013), Ross-Hellauer (2016a, 2016b) o P2PFoundation(2016), a partir del análisis de las definiciones presentes en la creciente literatura, han descrito las principales características de la revisión abierta, enriqueciendo así el debate sobre sus ventajas e inconvenientes. Se pueden señalar tres 
principales rasgos independientes de la evaluación abierta que están presentes en diferentes grados en las publicaciones que adoptan este modelo.

Identidades abiertas: al asignar los editores un manuscrito para su revisión los evaluadores conocen el nombre de los autores y, posteriormente los autores, al recibir el dictamen, sabrán la identidad de los evaluadores junto a sus comentarios. En algunos casos se da también una “interacción abierta”, es decir, los editores permiten y posibilitan la discusión directa entre evaluadores y autores.

- Informes abiertos: al finalizar el proceso editorial del trabajo se publica junto con el artículo los informes firmados de los evaluadores. Cabe señalar que en los últimos años han surgido plataformas abiertas e independientes que permiten desvincular las revisiones de los artículos publicados (PubPeer, Publons, Faculty of 1000, etc.).

- Participación abierta: en estos casos se invita al conjunto de la comunidad a formar parte del proceso de arbitraje. En ocasiones esto va unido a subir en abierto los trabajos en su versión inicial previa a la evaluación (existen para ello plataformas como arXiv) y/o dejar la posibilidad de una revisión por pares posterior a la publicación oficial del trabajo.

El modelo de evaluación abierta adoptado por Encrucijadas se basa, por el momento, en la primera de estas tres características: los evaluadores conocen la identidad de los autores firmantes del trabajo cuando reciben el manuscrito y los autores conocen la identidad y afiliación institucional de los evaluadores externos cuando reciben el dictamen. Es importante señalar el trascendental rol que debe desempeñar en este proceso abierto el comité editorial como garantía de arbitraje entre las partes y control de las redes de colegas y redes institucionales. La decisión final sobre los trabajos, como en la mayoría de publicaciones académicas, la tiene el comité editorial. Frente a los modelos ciegos, al conocerse las identidades se presupone mayor transparencia y honradez por parte de los evaluadores a la hora de aceptar o no la evaluación en función de una posible relación con los autores que no haya detectado el comité editorial. Esta fue la razón principal que animó a los editores de Encrucijadas a optar por el modelo de identidades abiertas para su proceso de evaluación externa. Junto a eso, las dificultades de derechos de autoría y la propia logística hacían que en el momento de puesta en marcha de la revista fuera inviable optar también por informes abiertos y/o participación abierta6. Además, en el caso de Encrucijadas, los editores realizan en una primera fase una preselección de los trabajos recibidos para catalogar cuáles pasan a la evaluación por pares según el cumplimiento de las normas y políticas de sección de la revista. Esto evita cargar en exceso e innecesariamente a los evaluadores con artículos claramente no aptos para su publicación, más aún cuando su identidad es pública.

6 Durante los primeros números se implementa un sistema piloto para fomentar la participación abierta, sin embargo la escasa red de contactos inicial de la propia revista y la exigencia de los plazos hacen descartar este modelo para la evaluación externa de los trabajos. 


\section{Un estudio de caso: la evaluación abierta de Encrucijadas}

Para contextualizar el proceso editorial de la revista de 2011 a 2016, la Tabla 1 recoge la actividad de los primeros seis años de Encrucijadas. La redacción de la revista ha recibido 236 trabajos, de los cuales se han publicado 81 (un 34,3\%) y se han rechazado el resto (el 65,7\%). Durante el periodo que se analiza (2011-2013) se publicaron un total de 37 trabajos científicos, con un enorme incremento en la recepción de artículos en el año 2013 (Tabla 1). Debido a ello, el índice de rechazo aumentó de un $41 \%$ en 2011 y un 55\% en 2012 a casi un 80\% en 2013. Tras ese pico, en los años posteriores se estabiliza el índice de rechazos entre un 57 y un $69 \%$.

Tabla 1: Número de trabajos (artículos y notas de investigación) recibidos, publicados y rechazados pre-evaluación y post-evaluación por pares. Encrucijadas, 2011-2016.

\begin{tabular}{|c|c|c|c|c|c|c|c|c|}
\hline & \multicolumn{2}{|c|}{ Publicados } & \multicolumn{2}{|c|}{ Rechazados post-evaluación } & \multicolumn{2}{|c|}{$\begin{array}{c}\text { Rechazados } \\
\text { pre-evaluación }\end{array}$} & \multicolumn{2}{l|}{ Recibidos } \\
\hline & Total & $\%$ & Total & $\%$ & Total & $\%$ & Total & $\%$ \\
\hline 2011 & 13 & 59,1 & 2 & 9,1 & 7 & 31,8 & 22 & 100 \\
\hline 2012 & 9 & 45,0 & 0 & 0,0 & 11 & 55,0 & 20 & 100 \\
\hline 2013 & 15 & 20,8 & 3 & 4,2 & 54 & 75,0 & 72 & 100 \\
\hline 2014 & 13 & 34,2 & 1 & 2,6 & 24 & 63,2 & 38 & 100 \\
\hline 2015 & 14 & 31,1 & 4 & 8,9 & 27 & 60,0 & 45 & 100 \\
\hline 2016 & 17 & 42,5 & 3 & 7,5 & 20 & 50,0 & 40 & 100 \\
\hline & & & & & & & & \\
\hline Total & 81 & 34,3 & 12 & 5,1 & 143 & 60,6 & 236 & 100 \\
\hline
\end{tabular}

Fuente: Elaboración propia a partir de datos del proceso editorial de Encrucijadas.

En total, entre 2011 y 2016 se han evaluado 93 manuscritos, de los que el $12,9 \%$ fueron rechazados tras los comentarios de los evaluadores externos. Las razones que hay detrás de este bajo porcentaje de rechazos son dos. Por un lado, el proceso de pre-selección interna es sumamente exigente, de modo que los manuscritos que pasan a evaluación ya presentan, a juicio del Comité Editorial, una calidad aceptable para ser publicados. De hecho, la mayor criba se produce en esa preselección, tal y como se constata en la Tabla 1, con un porcentaje de rechazo en esta fase del 60\%. Esta pre-selección, similar en otras muchas revistas de ciencias sociales, garantiza poder trabajar con un volumen razonable de manuscritos y no sobrecargar a los evaluadores con textos claramente no publicables. Por otro lado, es posible que las evaluaciones externas en un modelo de evaluación abierta se orienten en mayor medida a la crítica constructiva, de modo que tanto desde los evaluadores, los autores, como desde el Comité Editorial se recojan las revisiones como un instrumento de mejora que, en último término, redunda en la publicación de versiones muy mejoradas de los artículos. 
La comparativa con otras revistas aporta una perspectiva externa que puede ayudar a comprender nuestros datos. Por un lado, describimos las estadísticas de dos revistas indexadas en el Journal Citation Reports que elabora la empresa Thomson-Reuters: la Revista Internacional de Sociología (RIS) y la Revista Española de Investigaciones Sociológicas (REIS), ambas con revisión externa por pares ciegos. De 2011 a 2016, la RIS ha tenido un rechazo medio tras evaluación externa del 31\%, si bien con una tendencia descendente en el tiempo. La tendencia ha sido un aumento del rechazo en evaluación interna. Por su parte, en el año 2016 la REIS rechazó al 65\% en la selección previa y al 18\% tras evaluación externa. Por otro lado, tenemos las estadísticas de Teknokultura, revista con una concepción similar a la de Encrucijadas. En los años 2015 y 2016, únicos con estadísticas disponibles, el rechazo en selección previa fue del 78\% y el 23\%, respectivamente; en tanto, el rechazo tras revisión por pares fue del 28\% y el 17\%, respectivamente. Todos estos datos muestran que los índices de rechazo post-evaluación son generalmente bajos, si bien es cierto que en Encrucijadas son incluso más bajos, probablemente debido al volumen de artículos que se reciben y a los altos índices de rechazo en evaluación interna.

El número medio de evaluadores por artículo se incrementó de forma constante desde los primeros pasoso de la revista (Gráfico 1), superando a partir de 2014 los dos evaluadores por artículo, cifra mínima objetivo que se estableció desde la fundación de la revista para poder garantizar una evaluación objetiva y diversa. En estos seis años se ha contado con un total de 181 evaluadores externos que han participado en el modelo de arbitraje abierto de Encrucijadas, con una ligera masculinización (de cada 10 evaluadores, 6 han sido hombres y 4 mujeres). La Tabla 1 también muestra el porcentaje de artículos rechazados tras el proceso de evaluación. Conforme se va consolidando el proceso editorial se persigue el objetivo de que el número de trabajos disponibles para su publicación no sea un freno para el posible rechazo tras conocer el dictamen de los evaluadores externos. Esta razón, la necesidad de disponer de artículos para su publicación final durante los primeros años de existencia de la revista, y no tanto el modelo de evaluación abierta, está detrás de esas bajas tasas de rechazo post-evaluación. Entre 2011 y 2013, objeto de este estudio, se llevaron a cabo 66 evaluaciones externas a 41 artículos, 59\% de ellas realizadas por hombres, con una media de edad de 35,7 años, y un 41\% por mujeres, con una media de edad de 37,4 años.

Respecto a los autores, entre 2011 y 2016 han mandado sus colaboraciones 315 autores, de los cuales el 38\% pertenecían a instituciones de fuera de España. Se observa un importante sesgo entre hombres y mujeres: mientras que del total de trabajos recibidos un 56,5\% de los autores son hombres y un 43,2\% mujeres, entre los autores que finalmente publican sus trabajos el 66,7\% son hombres frente al 33,3\% de mujeres. En el periodo estudiado en el presente artículo publicaron 42 autores: un $69 \%$ fueron hombres y un $31 \%$ mujeres. Según los datos de la encuesta interna, la media de edad fue de 32,5 años entre los primeros y de 34,6 entre las segundas. 
Gráfico 1. Promedio anual de personas evaluadoras externas por artículo evaluado.

Encrucijadas, 2011-2016

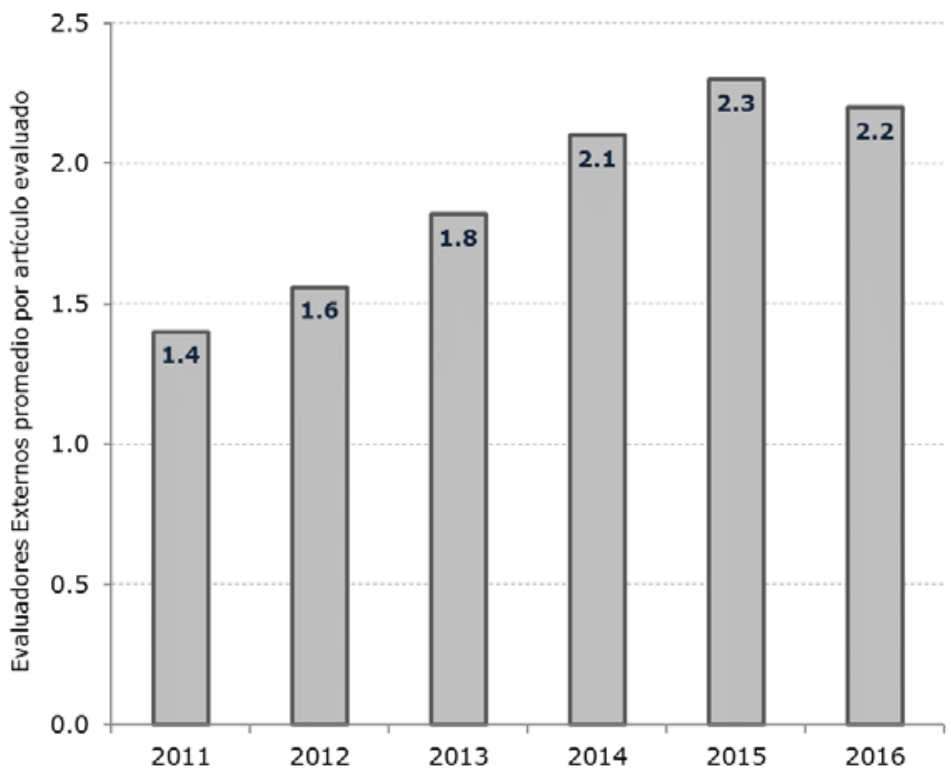

Fuente: Elaboración propia a partir de datos del proceso editorial de Encrucijadas.

\section{Datos y métodos}

\subsection{Encuesta}

Con el propósito de supervisión y evaluación de los procesos, el comité editorial lanzó durante el primer semestre de 2014 un cuestionario (anexo I) tanto a evaluadores como a autores que habían participado en Encrucijadas en los seis primeros números, de modo que tuviéramos información primaria para poder analizar la potencialidad y la viabilidad de los procesos de arbitraje abierto en general, y de Encrucijadas en particular. Asimismo, el cuestionario presentaba un módulo de preguntas sobre los procesos de evaluación por pares ciegos, de modo que pudiéramos establecer una comparativa entre ambos a partir de la experiencia de los sujetos que ya habían experimentado la evaluación abierta.

\subsection{Objetivos e hipótesis}

De una forma general, queríamos conocer las limitaciones y potencialidades de los diferentes modelos de evaluación. El objetivo era evaluar el proceso editorial de la revista, para lo que quisimos analizar la percepción y las actitudes de autores y evaluadores hacia el modelo de doble ciego y el modelo de la evaluación abierta. 
Nos planteamos tres hipótesis:

$H_{1} \equiv$ Las mujeres serán menos proclives al modelo de doble ciego.

Esta hipótesis descansa en la secular discriminación por género que las mujeres han tenido tradicionalmente en el ámbito científico. De esta manera, partimos de la hipótesis de que las mujeres serán más tendentes a una evaluación abierta que pueda visibilizar su aportación a la ciencia. Sin embargo, sí que éramos conscientes de que podría haber un efecto contrario derivado de la exposición pública del trabajo de una mujer en un espacio de dominación masculina. Por un lado, como autora, que se conociera su sexo podría incidir en prejuicios previos. Por otro lado, como evaluadora, podría tener repercusiones en la aceptación de críticas por parte de los autores masculinos.

$H_{2} \equiv$ La evaluación abierta será mejor recibida por autores que por evaluadores.

Tradicionalmente el proceso de doble ciego ha salvaguardado la identidad de los evaluadores, de modo que estos no temían hacer una revisión de índole destructivo o insustancial. Partimos de la idea de que los evaluadores querrían seguir amparándose en ese anonimato, de modo que serían menos proclives a la evaluación abierta. Frente a ellos, los autores la aceptarían más porque eso les garantizaría una mayor visibilidad, desde su parte, y conocer a otros expertos en la materia que trabajan, desde la de los evaluadores.

$H_{3} \equiv$ La evaluación abierta será mejor recibida cuanto menor sea la edad.

La socialización académica de las personas más jóvenes ha sido en un entorno más aperturista y con mayor nivel de transparencia en las publicaciones científicas, especialmente en revistas digitales de acceso abierto. El estudio de CruzCastro y Sanz-Menendez (2010) llega a la conclusión de que en España las trayectorias académicas más móviles (institucional, sectorial y geográficamente), significativamente más frecuentes en los jóvenes, no suponen una mejor inserción laboral debido a unas instituciones endogámicas, pero si apunta a importantes diferencias en términos de productividad y de formas de hacer en la práctica científica. Partimos así de la hipótesis de que las personas más jóvenes serán más favorables al modelo de evaluación abierta.

\subsection{Población y muestra}

El cuestionario se realizó con autores y evaluadores de los seis primeros números de la revista. Se pasó el cuestionario a 58 personas, de las cuales 27 habían enviado artículos y 37 habían colaborado en la evaluación. Dado que a algunos autores se les solicitó posteriormente colaborar como evaluadores, seis de las personas encuestadas eran al mismo tiempo autores y evaluadores. La tasa de respuesta fue alta: entre los autores alcanzó el 93,1\% y entre los evaluadores fue del $80 \%$. 


\subsection{Variables y métodos}

Se seleccionaron tres variables independientes dicotómicas: sexo (varón / mujer), edad ( $<35$ años, $\geq 35$ años) y tipo de contribución (autor / evaluador), para explicar algunos rasgos del proceso de evaluación de Encrucijadas y las opiniones ante los modelos de evaluación abierta y cerrada. Las variables dependientes que se tomaron fueron las ocho siguientes variables dicotómicas categorizadas como no/sí, que se dividen en dos grupos referidos al proceso de evaluación de doble ciego.

\subsubsection{Aspectos positivos del proceso de doble ciego}

- Se elimina de los artículos cualquier pista que ayude a identificar a los autores.

- Los evaluadores hacen la revisión de forma más justa.

- Los autores reciben mejor los comentarios al no conocer la identidad de los evaluadores.

- Los artículos que pasan por un sistema de doble ciego mejoran siempre su calidad frente a otros modelos de arbitraje.

\subsubsection{Aspectos negativos del proceso de doble ciego}

- Ocultar la identidad no evita el sesgo ni varía la evaluación de los revisores ni la forma de recibir los comentarios de los autores.

- Elaborar una versión anonimizada complica la redacción del manuscrito y finalmente no ayuda a mejorar la calidad del artículo.

- Es fácil identificar el autor o al menos la institución o el género, más aún en un campo pequeño.

- Revisiones normales y de doble ciego no presentaban diferencias en cuanto a las recomendaciones.

El hecho de preguntar por este modelo de evaluación en lugar de hacerlo para el modelo abierto presente en la revista reside en tres motivos. Primero, el proceso de doble ciego es más conocido por autores y evaluadores, de modo que la información recogida sería más fiable o, al menos, basada en una mayor cantidad de evidencias. Segundo, no teníamos información directa sobre el doble ciego de autores o evaluadores que sí hubieran participado en un proceso de evaluación abierta; de esta manera se podía combinar una muestra novedosa con los estudios clásicos previos. Y tercero, los datos serían complementarios a las percepciones sobre la evaluación abierta, lo que permitiría una comparación más transparente.

En el caso de los modelos de evaluación abierta, la recogida de información se realizó a través de dos tipos de preguntas. Por un lado, se hizo una pregunta general sobre si ese tipo de procesos aportaba una mayor transparencia a las evaluaciones. Por otro lado, se estableció un módulo de preguntas sobre el modelo de evaluación 
abierta específico de Encrucijadas, si bien con una gran orientación al conjunto de este tipo de modelos.

- (Para autores)

Usted como autor, ¿¿en qué medida modificó su texto en función de los comentarios tras la evaluación por pares?

- (Para evaluadores)

Usted como evaluador, ¿¿cree que su revisión y recomendaciones se vio modificada al ser abierto el proceso?

- (Para autores y evaluadores)

La posibilidad de ver el nombre de los autores/evaluadores aporta transparencia al proceso de evaluación.

Además de dejar visibles los nombres para autores y evaluadores, el proceso de evaluación ganaría calidad dejando visibles los comentarios de evaluación una vez termina el proceso de arbitraje.

El motivo para referirnos de forma concreta a la revista residió en que queríamos que basaran sus respuestas en la experiencia con la revista, de modo que, además de conseguir una evaluación de nuestro funcionamiento interno, podríamos obtener información que pudiéramos comparar con la anteriormente recogida para el modelo de doble ciego. Es necesario apuntar una limitación del cuestionario: la forma de preguntar por cada tipo de modelo fue diferente, de modo que es posible que se creara algún tipo de sesgo en la comparabilidad. No obstante, dados los resultados no parece que el estilo de preguntas produjera un error lo suficientemente sustantivo como para tergiversar las conclusiones alcanzadas.

Para establecer si en cada uno de los aspectos analizados había diferencias entre las categorías de cada una de las tres variables independientes se realizó un análisis a través de tablas de contingencia 2x2, de modo que pudiéramos establecer, en el caso de que hubiera asociación, el odds ratio. Asimismo, para profundizar en la posible importancia del género y del tipo de actor, se realizaron análisis estratificados por esas dos variables.

\section{Resultados}

\subsection{Opinión sobre el método de Doble Ciego}

En primer lugar, la Tabla 2 muestra los porcentajes de acuerdo con las diferentes afirmaciones tanto a favor como en contra del modelo de evaluación conocido como doble ciego que en la actualidad es el mayoritario en las publicaciones científicas. Se diferencian dos grupos: personas autoras y personas evaluadoras, y dentro de cada uno de ellos se estratifica por sexo. En general, los porcentajes son diferentes tanto por tipo de contribución como por sexo, si bien debemos ser cautos dado el reducido tamaño de la muestra. Tanto autoras como evaluadoras señalan como principal ventaja del doble ciego la forma más justa de evaluación que hacen los pares, ventaja que es más señalada por mujeres que por hombres en ambas figuras, pero especialmente entre los evaluadores. Del lado negativo destaca el hecho de 
que el doble ciego no evita el probable sesgo en la evaluación, ni la posible identificación de los autores (instituciones o su género) y no varía la forma de recibir los comentarios.

Para profundizar en las diferencias porcentuales, se realiza un análisis estadístico con tablas de contingencia. Se ha analizado si existe relación entre las variables dependientes que aparecen en las tablas y tres variables independientes: sexo (varón / mujer) (Tabla 3), edad (<35 / $\geq 35$ ) (Tabla 4) y ser autor / evaluador (Tabla 5). En ninguno de los casos hay relación, de modo que encontramos que las variables que caracterizan al proceso de evaluación abierta son independientes del sexo, la edad y el rol que ha desempeñado cada actor (Tabla 2). Por lo tanto, con el análisis estadístico bivariante no podemos corroborar ninguna de las hipótesis planteadas, algo que veremos que, al menos parcialmente, se puede matizar mediante el análisis de las respuestas a las preguntas abiertas.

En cambio, al realizar una tabla de contingencia con las variables independientes “autor” (no/sí) y “evaluador” (no/sí) estratificada por sexo, sólo se encuentra relación en una ocasión. En la variable dependiente "Es fácil identificar el autor o al menos la institución o el género, más aún en un campo pequeño (no/sí)” la relación en el estrato "varón” es significativa entre los autores (phi = -,378; p = ,038; OR = ,194). Es decir, entre los varones que no son autores hay menos probabilidad de encontrar personas que no crean que es fácil la identificación por alguno de esos rasgos (tablas no mostradas).

Tabla 2. Porcentaje de acuerdo con las afirmaciones sobre el doble ciego de autores y evaluadores según sexo.

\begin{tabular}{|c|c|c|c|c|c|}
\hline & & \multicolumn{2}{|c|}{ Autor } & \multicolumn{2}{|c|}{ Evaluador } \\
\hline & & \multicolumn{4}{|c|}{ (H=Hombre/M=Mujer) } \\
\hline & & $\mathrm{H}$ & $\mathrm{M}$ & $\mathrm{H}$ & $\mathrm{M}$ \\
\hline \multirow{5}{*}{$\begin{array}{l}\text { Aspectos } \\
\text { positivos } \\
\text { del doble } \\
\text { ciego }\end{array}$} & $\begin{array}{l}\text { Se elimina de los artículos cualquier pista que } \\
\text { ayude a identificar a los autores (no/sí) }\end{array}$ & 43,8 & 30,0 & 42,9 & 14,3 \\
\hline & $\begin{array}{l}\text { Los autores reciben mejor los comentarios al } \\
\text { no conocer la identidad de los evaluadores } \\
\text { (no/sí) }\end{array}$ & 25,0 & 40,0 & 28,6 & 57,1 \\
\hline & $\begin{array}{l}\text { Los evaluadores hacen la revisión de forma } \\
\text { más justa (no/sí) }\end{array}$ & 56,3 & 60,0 & 57,1 & 78,6 \\
\hline & $\begin{array}{l}\text { Los artículos que pasan por un sistema de } \\
\text { doble ciego mejoran siempre su calidad frente } \\
\text { a otros modelos de arbitraje (no/sí) }\end{array}$ & 31,3 & 0,0 & 23,8 & 28,6 \\
\hline & Ningún aspecto positivo & 0,0 & 10,0 & 9,5 & 7,1 \\
\hline \multirow{3}{*}{$\begin{array}{l}\text { Aspectos } \\
\text { negativos } \\
\text { del doble } \\
\text { ciego }\end{array}$} & $\begin{array}{l}\text { Es fácil identificar el autor o al menos la insti- } \\
\text { tución o el género, más aún en un campo pe- } \\
\text { queño (no/sí) }\end{array}$ & 20,0 & 55,6 & 45,0 & 38,5 \\
\hline & $\begin{array}{l}\text { Elaborar una versión anonimizada complica la } \\
\text { redacción del manuscrito y finalmente no } \\
\text { ayuda a mejorar la calidad del artículo (no/sí) }\end{array}$ & 6,7 & 22,2 & 25,0 & 7,7 \\
\hline & Revisiones normales y de doble ciego no pre- & 20,0 & 0,0 & 25,0 & 7,7 \\
\hline
\end{tabular}




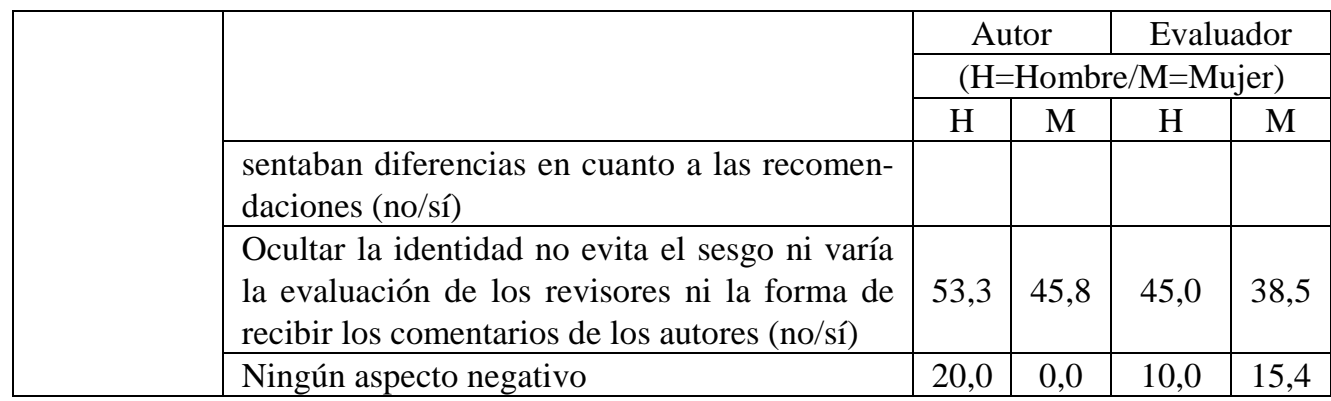

Fuente: Elaboración propia.

Tabla 3. Tablas de contingencia. Variable independiente: sexo (varón/mujer).

\begin{tabular}{|l|c|c|c|}
\hline & \multicolumn{3}{|c|}{ Sexo (varón/mujer) } \\
\cline { 2 - 4 } & Phi & OR & p \\
\hline $\begin{array}{l}\text { Se elimina de los artículos cualquier pista que ayude a identi- } \\
\text { ficar a los autores (no/sí) }\end{array}$ &,- 186 &, 427 & 0,164 \\
\hline $\begin{array}{l}\text { Los autores reciben mejor los comentarios al no conocer la } \\
\text { identidad de los evaluadores (no/sí) }\end{array}$ &, 211 & 2,444 &, 211 \\
\hline Los evaluadores hacen la revisión de forma más justa (no/sí) &, 138 & 1,842 &, 301 \\
\hline $\begin{array}{l}\text { Los artículos que pasan por un sistema de doble ciego mejoran } \\
\text { siempre su calidad frente a otros modelos de arbitraje (no/sí) }\end{array}$ &,- 082 &, 658 &, 539 \\
\hline $\begin{array}{l}\text { Es fácil identificar el autor o al menos la institución o el géne- } \\
\text { ro, más aún en un campo pequeño (no/sí) }\end{array}$ &, 088 & 1,439 &, 523 \\
\hline $\begin{array}{l}\text { Elaborar una versión anonimizada complica la redacción del } \\
\text { manuscrito y finalmente no ayuda a mejorar la calidad del } \\
\text { artículo (no/sí) }\end{array}$ &,- 025 &, 867 &, 857 \\
\hline $\begin{array}{l}\text { Revisiones normales y de doble ciego no presentaban diferen- } \\
\text { cias en cuanto a las recomendaciones (no/sí) }\end{array}$ &,- 210 &, 208 &, 130 \\
\hline $\begin{array}{l}\text { Ocultar la identidad no evita el sesgo ni varía la evaluación de } \\
\text { los revisores ni la forma de recibir los comentarios de los } \\
\text { autores (no/sí) }\end{array}$ &,- 102 &, 656 &, 463 \\
\hline
\end{tabular}

Fuente: Elaboración propia. 
Tabla 4. Tablas de contingencia. Variable independiente: edad ( $<35$ años / $\geq 35$ años)

\begin{tabular}{|c|c|c|c|}
\hline & \multicolumn{3}{|c|}{$\operatorname{Edad}(<35 / \geq 35$ años $)$} \\
\hline & Phi & OR & $\mathrm{p}$ \\
\hline $\begin{array}{l}\text { Se elimina de los artículos cualquier pista que ayude a identifi- } \\
\text { car a los autores (no/sí) }\end{array}$ & ,170 & 2,083 & ,208 \\
\hline $\begin{array}{l}\text { Los autores reciben mejor los comentarios al no conocer la } \\
\text { identidad de los evaluadores (no/sí) }\end{array}$ & ,106 & 1,568 & ,431 \\
\hline Los evaluadores hacen la revisión de forma más justa (no/sí) & 070 & 1,364 & 606 \\
\hline $\begin{array}{l}\text { Los artículos que pasan por un sistema de doble ciego mejoran } \\
\text { siempre su calidad frente a otros modelos de arbitraje (no/sí) }\end{array}$ & ,168 & 2,320 & ,212 \\
\hline $\begin{array}{l}\text { Es fácil identificar el autor o al menos la institución o el género, } \\
\text { más aún en un campo pequeño (no/sí) }\end{array}$ &,- 085 & ,703 & ,543 \\
\hline $\begin{array}{l}\text { Elaborar una versión anonimizada complica la redacción del } \\
\text { manuscrito y finalmente no ayuda a mejorar la calidad del ar- } \\
\text { tículo (no/sí) }\end{array}$ &,- 142 & ,421 & 311 \\
\hline $\begin{array}{l}\text { Revisiones normales y de doble ciego no presentaban diferen- } \\
\text { cias en cuanto a las recomendaciones (no/sí) }\end{array}$ & ,065 & 1,500 & ,640 \\
\hline $\begin{array}{l}\text { Ocultar la identidad no evita el sesgo ni varía la evaluación de } \\
\text { los revisores ni la forma de recibir los comentarios de los auto- } \\
\text { res (no/sí) }\end{array}$ &,- 038 & ,857 & ,788 \\
\hline
\end{tabular}

Fuente: Elaboración propia.

Tabla 5. Tablas de contingencia. Variable independiente: contribución (autor / evaluador).

\begin{tabular}{|l|c|c|c|}
\hline & \multicolumn{3}{|c|}{ Autor / evaluador } \\
\hline & Phi & OR & p \\
\hline $\begin{array}{l}\text { Se elimina de los artículos cualquier pista que ayude a identi- } \\
\text { ficar a los autores (no/sí) }\end{array}$ &,- 072 &, 727 &, 607 \\
\hline $\begin{array}{l}\text { Los autores reciben mejor los comentarios al no conocer la } \\
\text { identidad de los evaluadores (no/sí) }\end{array}$ &, 118 & 1,667 &, 401 \\
\hline Los evaluadores hacen la revisión de forma más justa (no/sí) &, 072 & 1,375 &, 607 \\
\hline $\begin{array}{l}\text { Los artículos que pasan por un sistema de doble ciego mejoran } \\
\text { siempre su calidad frente a otros modelos de arbitraje (no/sí) }\end{array}$ &, 112 & 1,826 &, 423 \\
\hline $\begin{array}{l}\text { Es fácil identificar el autor o al menos la institución o el géne- } \\
\text { ro, más aún en un campo pequeño (no/sí) }\end{array}$ &, 078 & 1,375 &, 595 \\
\hline $\begin{array}{l}\text { Elaborar una versión anonimizada complica la redacción del } \\
\text { manuscrito y finalmente no ayuda a mejorar la calidad del } \\
\text { artículo (no/sí) }\end{array}$ &, 101 & 1,848 &, 488 \\
\hline $\begin{array}{l}\text { Revisiones normales y de doble ciego no presentaban diferen- } \\
\text { cias en cuanto a las recomendaciones (no/sí) }\end{array}$ &, 144 & 3,000 &, 325 \\
\hline $\begin{array}{l}\text { Ocultar la identidad no evita el sesgo ni varía la evaluación de } \\
\text { los revisores ni la forma de recibir los comentarios de los } \\
\text { autores (no/sí) }\end{array}$ & $-0,045$ &, 833 &, 760 \\
\hline
\end{tabular}

Fuente: Elaboración propia. 
En cuanto al doble ciego, el estudio saco a la luz actitudes divididas entre aspectos positivos y negativos. Hubo quienes encontraban en este modelo una mejor opción que favorece mejores comentarios por parte de los evaluadores y evaluadoras.

"me parece mejor la evaluación por el método del doble ciego, permite que el texto sea valorado únicamente a partir de su calidad y contenidos, sin que entren en juego ulteriores consideraciones. " [Cuestionario nº6. Autor. Mujer. 34 años]

Sin embargo, se tendía a reconocer que tampoco el doble ciego garantiza el anonimato en un campo como el de las ciencias sociales y que, por tanto, no se evita el sesgo, tanto en la propia evaluación como en la forma de hacer y recibir evaluaciones.

Así mismo, se criticaba de este modelo la posibilidad de utilizar el anonimato para hacer evaluaciones extremadamente críticas y haciendo uso incluso de mala praxis.

“Destructivismo amparado en el anonimato.” [Cuestionario n7. Evaluador. Varón. 32 años]

“Conocer el nombre del autor del artículo puede condicionar la evaluación de algunos evaluadores” [Cuestionario nº10. Autor. Varón. 45 años]

En este caso, y en contraste con lo que apuntaba el análisis estadístico, pudimos confirmar la hipótesis primera que nos planteamos pues se pudo apreciar que las mujeres confían ligeramente menos en la ventaja del anonimato que supone el doble ciego. Quizás, pensamos, por que sea más fácil de reconocer el género del autor.

En el caso de la evaluación abierta se percibe de igual modo una actitud dividida. Si bien se aprecia un potencial en este modelo al considerar una mayor seriedad de las evaluaciones, se resalta la idea de que éstas también pueden formularse de una forma más ambigua.

“Preferible: mejora las evaluaciones y enriquece el contacto académico." [Cuestionario $n^{\circ} 7$. Evaluador. Varón. 32 años]

Con respecto a nuestra hipótesis, tal y como se veía con el análisis de tablas de contingencia, no podemos afirmar que existieran diferencias significativas entre autores y evaluadores, aunque los evaluadores están más preocupados por la comunicación entre las partes en el proceso.

\subsection{Opinión sobre el método de Evaluación Abierta}

En segundo lugar, se analiza las opiniones hacia los modelos de evaluación abierta (open peer review), en especial indagando sobre su experiencia en Encrucijadas. Una aproximación general sobre la visión hacia los modelos abiertos de evaluación se mide con el grado de acuerdo hacia la afirmación de que este tipo de evaluación aporta mayor transparencia (Tabla 6 y Gráfico 2). 
Tabla 6. Frecuencias de las preguntas referidas al proceso de evaluación abierta por sexo y tipo de contribución.

\begin{tabular}{|c|c|c|c|c|}
\hline \multirow{2}{*}{$\begin{array}{l}\text { Autores y } \\
\text { evaluadores }\end{array}$} & & \multirow[t]{2}{*}{ Total } & \multicolumn{2}{|c|}{$\begin{array}{c}\text { (H=Hombre/ } \\
\text { M=Mujer })\end{array}$} \\
\hline & & & $\mathrm{H}$ & $\mathrm{M}$ \\
\hline \multirow{5}{*}{$\begin{array}{l}\text { La posibilidad de ver el nom- } \\
\text { bre de los autores/evaluadores } \\
\text { aporta transparencia al proce- } \\
\text { so de evaluación }\end{array}$} & Completamente de acuerdo & 18,2 & 15,2 & 22,7 \\
\hline & De acuerdo & 29,1 & 27,3 & 31,8 \\
\hline & $\begin{array}{l}\text { Ni en acuerdo ni en } \\
\text { desacuerdo }\end{array}$ & 12,7 & 15,2 & 9,1 \\
\hline & En desacuerdo & 38,2 & 42,4 & 31,8 \\
\hline & $\begin{array}{l}\text { Completamente en } \\
\text { desacuerdo }\end{array}$ & 1,8 & 0,0 & 4,5 \\
\hline \multirow{5}{*}{$\begin{array}{l}\text { Además de dejar visibles los } \\
\text { nombres para autores y eva- } \\
\text { luadores, el proceso de eva- } \\
\text { luación ganaría calidad } \\
\text { dejando visibles los comenta- } \\
\text { rios de evaluación una vez } \\
\text { termina el proceso de arbitra- } \\
\text { je. }\end{array}$} & Completamente de acuerdo & 17,3 & 20,0 & 13,6 \\
\hline & De acuerdo & 32,7 & 23,3 & 45,5 \\
\hline & $\begin{array}{l}\text { Ni en acuerdo ni en } \\
\text { desacuerdo }\end{array}$ & 25,0 & 26,7 & 22,7 \\
\hline & En desacuerdo & 25,0 & 30,0 & 18,2 \\
\hline & $\begin{array}{l}\text { Completamente en } \\
\text { desacuerdo }\end{array}$ & 0,0 & 0,0 & 0,0 \\
\hline \multicolumn{5}{|l|}{ Autores } \\
\hline \multirow{3}{*}{$\begin{array}{l}\text { Usted como autor, ¿en qué } \\
\text { medida modificó su texto en } \\
\text { función de los comentarios } \\
\text { tras la evaluación por pares? }\end{array}$} & $\begin{array}{l}\text { Realicé cambios sustanciales } \\
\text { en el artículo }\end{array}$ & 30,8 & 37,5 & 20,0 \\
\hline & $\begin{array}{l}\text { Realicé cambios menores en } \\
\text { el artículo }\end{array}$ & 61,5 & 50,0 & 80,0 \\
\hline & No realicé ningún cambio & 37,7 & 12,5 & 0,0 \\
\hline \multicolumn{5}{|l|}{ Evaluadores } \\
\hline \multirow{4}{*}{$\begin{array}{l}\text { Usted como evaluador, ¿cree } \\
\text { que su revisión y recomenda- } \\
\text { ciones se vio modificada al ser } \\
\text { abierto el proceso? }\end{array}$} & $\begin{array}{l}\text { El autor revisó convenien- } \\
\text { temente el texto en la línea } \\
\text { indicada por los comentarios }\end{array}$ & 20,7 & 18,8 & 23,1 \\
\hline & $\begin{array}{l}\text { El autor revisó ligeramente } \\
\text { el texto, aunque no siguió } \\
\text { todas las indicaciones seña- } \\
\text { ladas }\end{array}$ & 29,6 & 25,0 & 30,8 \\
\hline & $\begin{array}{l}\text { El autor hizo caso omiso de } \\
\text { las recomendaciones }\end{array}$ & 3,4 & 6,3 & 0,0 \\
\hline & $\begin{array}{l}\text { No revisé el estado final del } \\
\text { texto, no puedo valorar las } \\
\text { modificaciones realizadas }\end{array}$ & 48,3 & 50,0 & 46,2 \\
\hline
\end{tabular}

Fuente: Elaboración propia. 
Gráfico 2. Grado de acuerdo de autores y evaluadores con la afirmación "la posibilidad de ver el nombre de los autores/evaluadores aporta transparencia al proceso de evaluación”, según sexo.

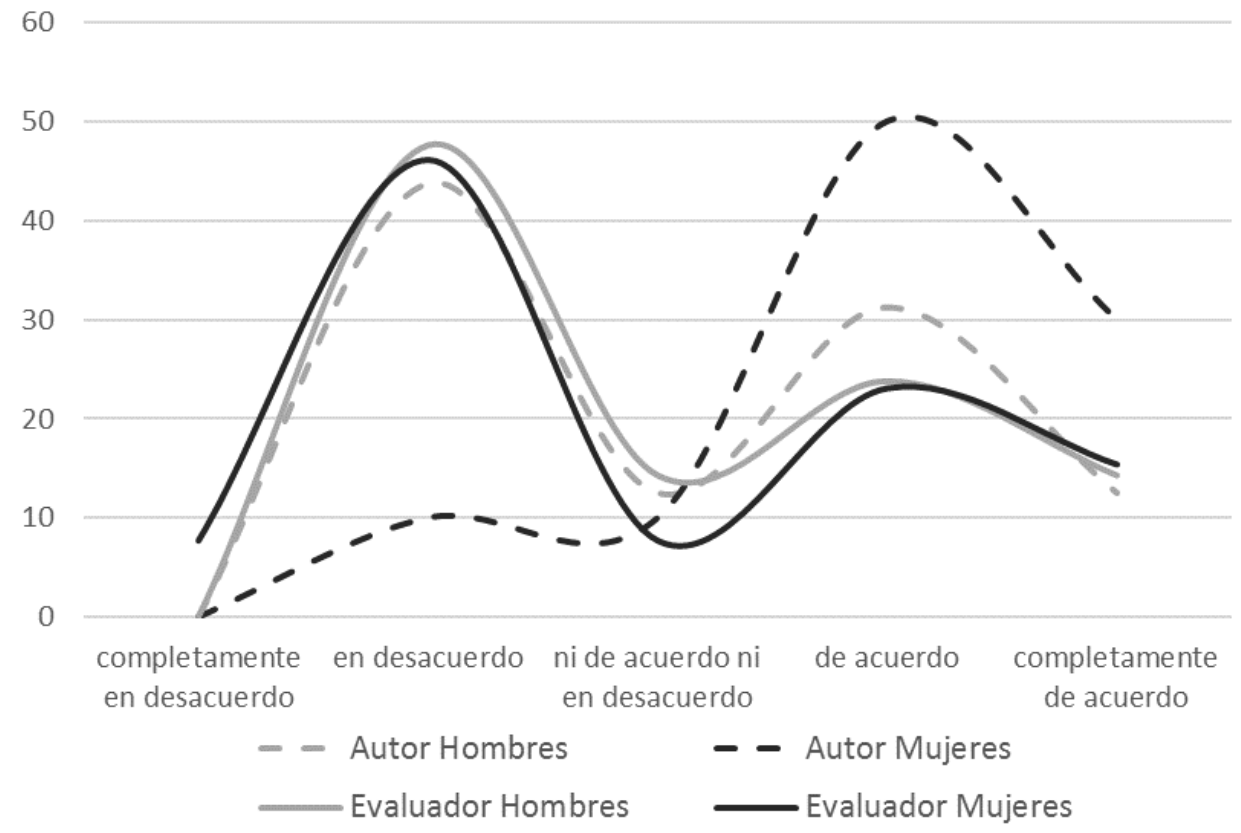

Fuente: Elaboración propia.

Los resultados muestran tres tendencias reseñables. Primero, una división, con peso muy similar, entre los que están de acuerdo y desacuerdo con la mayor transparencia de la evaluación abierta. Segundo, los autores están más de acuerdo con esa afirmación mientras que una mayoría de los evaluadores están en desacuerdo o completamente desacuerdo. Tercero, resulta especialmente interesante observar que entre las autoras mujeres el $80 \%$ está de acuerdo o completamente de acuerdo con esa mayor trasparencia del open peer review, tendencia muy dispar a la del resto del grupo y que puede explicarse por la sensación de incapacidad de evitar un sesgo de género por parte de los modelos de doble ciego.

La Tabla 7 muestra cuáles fueron las percepciones y actitudes hacia la evaluación abierta de Encrucijadas tanto de evaluadores como de autores. Hemos diferenciado entre elementos positivos hacia el modelo de evaluación abierta y las desventajas de ese modelo frente al doble ciego. En ambos grupos de contribuyentes se valoró positivamente la existencia de una comunicación más directa que ayudaba a incrementar la calidad de los manuscritos y la responsabilidad como garantes de la excelencia académica. Frente a ello, tanto evaluadores como autores apuntaron que, sin embargo, sí que podría producirse una mayor prudencia en las revisiones, lo que podría quitar contundencia o profundidad en las críticas. Por tanto, tanto en las ventajas como los inconvenientes no se observa una brecha entre autores y evaluadores, aunque sí una diferente forma de expresar una misma opi- 
nión. Por ejemplo, mientras los autores señalan que las evaluaciones al ser abiertas “son más prudentes”, los evaluadores hablan de “limitación de las críticas”.

Tabla 7. Percepción y actitudes hacia la evaluación abierta

\begin{tabular}{|c|c|c|}
\hline & $\begin{array}{l}\text { - Elementos positivos de la eva- } \\
\text { luación abierta }\end{array}$ & $\begin{array}{l}\text { - Desventajas de la evaluación } \\
\text { abierta frente al doble ciego }\end{array}$ \\
\hline \multirow{4}{*}{ 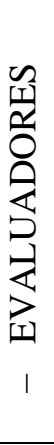 } & $\begin{array}{l}\text { - Comunicación más directa, } \\
\text { enriquece contexto académico. }\end{array}$ & - Puede crear resentimiento. \\
\hline & - Aporta transparencia. & $\begin{array}{l}\text { - Críticas más ambiguas o be- } \\
\text { nevolentes. Se limita la críti- } \\
\text { ca. }\end{array}$ \\
\hline & $\begin{array}{l}\text { - Actitud académica más res- } \\
\text { ponsable y comprometida. } \\
\text { Evaluaciones más serias. }\end{array}$ & $\begin{array}{l}\text { - Condicionamiento por filia- } \\
\text { ciones personales en caso de } \\
\text { que se conozcan. }\end{array}$ \\
\hline & $\begin{array}{l}\text { - Evita abusos y evaluaciones } \\
\text { destructivas. }\end{array}$ & - Menor objetividad \\
\hline \multirow{3}{*}{ 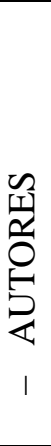 } & $\begin{array}{l}\text { - Lógica cercana de conoci- } \\
\text { miento situado ("más cercano, } \\
\text { abierto y humano”). Si cono- } \\
\text { ces quién te evalúa es más } \\
\text { sencillo valorar sus opiniones. }\end{array}$ & $\begin{array}{l}\text { - El texto es valorado única- } \\
\text { mente por su calidad y no en- } \\
\text { tra en juego nada más. }\end{array}$ \\
\hline & $\begin{array}{l}\text { - Comunicación más óptima } \\
\text { que en el doble ciego. }\end{array}$ & - Evaluaciones más prudentes. \\
\hline & $\begin{array}{l}\text { - Los evaluadores son más res- } \\
\text { ponsables, evaluaciones más } \\
\text { sólidas. }\end{array}$ & $\begin{array}{l}\text { - La evaluación abierta puede } \\
\text { predisponer más al evalua- } \\
\text { dor. }\end{array}$ \\
\hline
\end{tabular}

Fuente: Elaboración propia.

Estos resultados, para el caso de Encrucijadas, concuerdan con estudios previos. El trabajo de van Rooyen et al (1999), para el campo de la medicina, ya mostraba que las identidades abiertas en la evaluación por pares no tienen un efecto diferencial sobre la calidad de la revisión ni sobre la recomendación relativa a la publicación o no del manuscrito. Más recientemente, un estudio piloto llevado a cabo por Elsevier para valorar el impacto en el proceso de evaluación de conocer la identidad de los autores y la publicación posterior de los dictámenes (Mehmani, 2016) concluye precisamente que la mayor trasparencia que supone el open peer review no sólo no tienen un efecto sobre la decisión recomendada sino que un tercio de los editores consultados afirman que este modelo deriva en una mejora en la calidad general de los informes de revisión recibidos y un 70\% de los editores consideran que los dictámenes de los pares externos en los casos de evaluación abierta y posterior publicación de las evaluaciones se traduce en una mayor profundidad y un tono más constructivo para los autores puedan mejorar la calidad de sus textos. 


\section{Conclusiones}

Encrucijadas ha contado con una evolución positiva de recepción de artículos en los años que se estudiaron y que ha continuado hasta el presente, lo que ha permitido una mejor preselección de los trabajos recibidos y la instauración del arbitraje por pares de una forma más sólida y mejorando niveles de calidad. Los resultados de este estudio muestran la complejidad de opiniones y actitudes hacia los distintos modelos de evaluación, pero muestran cómo se abre un espacio para desarrollar y consolidar los modelos de evaluación abierta una vez se ha corroborado que no se considera significativamente de mejor o peor calidad y resultado que los modelos de doble ciego.

Las hipótesis que se habían planteado en un principio no se han corroborado mediante el análisis estadístico, lo que muestra la complejidad de la medición de los tipos de evaluación, a lo que se le añade la limitación de un bajo tamaño muestral. No obstante, la inclusión de preguntas abiertas en el cuestionario sí que ha permitido conocer los matices que, finalmente, introducen el elemento que confirma parcialmente las hipótesis iniciales. El propio contexto de la revista debe ser tenido en cuenta a la hora de poner en valor estos resultados. Por un lado, se han analizado los primeros años de publicación de la revista, apuntando ya los resultados a un cambio paulatino en la medida en que la consolidación de la publicación puede ir modificando el papel que juega este modelo de evaluación; por ejemplo, el incremento de los artículos rechazados tras los dictámenes de los pares externos. Por otro lado, el propio enfoque de Encrucijadas como revista, con un planteamiento crítico y con una media de edad joven (tanto de autores, como de evaluadores y editores) abre una oportunidad para que un modelo alternativo de evaluación se abra camino.

Los procesos de evaluación aún tienen muchos grandes retos por delante. Primero, investigadores y revistas nos encontramos inmersos en un sistema que ha sido tergiversado y mercantilizado por las grandes empresas editoriales, imponiendo sistemas de medición que, en ocasiones, nada tienen que ver con la calidad ni de las revistas, ni de los artículos, ni del proceso de evaluación. Segundo, las exigencias del actual modelo científico y de promoción académica han hecho que el volumen de artículos que se reciben se haya incrementado exponencialmente, lo que crea dificultades en la organización de los consejos de redacción -especialmente en aquellos que no tienen puestos profesionales o asistencia-, en la búsqueda de evaluadores y en la obtención de evaluaciones exhaustivas. Tercero, a colación de los anteriores, son pocas las revistas que ofrecen algún tipo de remuneración a unos evaluadores que ya están sobrecargados por sus obligaciones académicas. Sin embargo, esas empresas se lucran posteriormente con el acceso cerrado o la venta de artículos, con las suscripciones individuales o institucionales, o con el cobro de gastos editoriales. Y cuarto, la combinación del actual sistema de clasificación de las revistas y del sistema de promoción académica está generando un incremento de la brecha entre aquellas revistas que están incluidas en índices considerados más prestigiosos y aquellas que se encuentran fuera de ese circuito.

El futuro, aunque incierto, parece caminar hacia una diversidad de modelos, donde la propia cultura en ascenso del Open Access de un empuje definitivo a modelos alternativos de evaluación científica. Hemos observado como durante la úl- 
tima década un sinfín de revistas, especialmente en el campo de las ciencias sociales, han ido adoptando el acceso abierto. ¿Este cambio puede llegar a los modelos de evaluación? En este sentido, uno de los retos futuros que se plantea Encrucijadas es desarrollar un modelo piloto que permita sumar a la identidad abierta en las evaluaciones, el poner en abierto junto a los artículos los informes y dictámenes de los evaluadores. Estos informes abiertos suponen una enorme potencialidad docente creando bancos de evaluaciones pero a la vez presentan importante limitaciones que también pudieran condicionar las evaluaciones reforzando aspectos negativos que ya señalan tanto autores como evaluadores como principales inconvenientes de los modelos abiertos.

\section{Referencias bibliográficas}

Cruz Castro, L., \& Sanz Menéndez, L. (2010). Endogamia, productividad y carreras académicas. Recuperado a partir de https://digital.csic.es/handle/10261/24401

Csiszar, A. (2016, mayo 3). La revisión por pares (peer review) ha sido problemática desde sus comienzos [Blog]. Recuperado 18 de junio de 2017, a partir de http://www.sinpermiso.info/textos/la-revision-por-pares-peer-review-ha-sidoproblematica-desde-sus-comienzos

Ford, E. (2013). Defining and Characterizing Open Peer Review: A Review of the Literature. Library Faculty Publications and Presentations. https://doi.org/10.3138/jsp.44-4001

Mehmani, B. (2016, septiembre 22). Is open peer review the way forward? Recuperado 18 de junio de 2017, a partir de https://www.elsevier.com/reviewersupdate/story/innovation-in-publishing/is-open-peer-review-the-way-forward

P2PFoundation. (s. f.). Open Peer Review. En P2PFoundation Wiki. Recuperado a partir de https://wiki.p2pfoundation.net/Open_Peer_Review

Prado Arreaza, C., Velasco Ortuño, S., González Padial, V., \& Sanromán Rodríguez, T. (s. f.). Peer Review¿Garantía de la calidad de la investigación científica? Recuperado a partir de http://www.ugr.es/ setchift/docs/peer_review.pdf

Rooyen, S. van, Godlee, F., Evans, S., Black, N., \& Smith, R. (1999). Effect of open peer review on quality of reviews and on reviewers9recommendations: a randomised trial. BMJ, 318(7175), 23-27. https://doi.org/10.1136/bmj.318.7175.23

Ross-Hellauer, T. (2016a, octubre 30). Defining Open Peer Review: Part One - Competing Definitions. Recuperado 18 de junio de 2017, a partir de https://blogs.openaire.eu/?p=1371

Ross-Hellauer, T. (2016b, noviembre 2). Defining Open Peer Review: Part Two - Seven Traits of OPR: OpenAIRE blog [Blog]. Recuperado 18 de junio de 2017, a partir de https://blogs.openaire.eu/?p=1410 


\section{Anexo I: Cuestionario a personas autoras y evaluadoras.}

\section{A. SOCIODEMOGRÁFICAS}

1. Tipo de colaboración

- Autor

- Evaluador

- Otros (lector, suscriptor, etc.)

2. Sexo

- Mujer

- Varón

\section{Edad}

\section{B. MODELOS DE EVALUACIÓN}

En primer lugar, nos gustaría preguntarle por su opinión acerca del modelo de evaluación conocido como el doble ciego (double blind peer review), el modelo más extendido en las revistas científicas. Nos gustaría valorar ventajas e inconvenientes del mismo, y si lo creen conveniente, recoger críticas personales.

En las siguientes dos cuestiones, marque aquellas afirmaciones con las que esté de acuerdo.

\section{Aspectos positivos del método del doble ciego}

- Se elimina de los artículos cualquier pista que ayude a identificar a los autores

- Los autores reciben mejor los comentarios al no conocer la identidad de los evaluadores

- Los evaluadores hacen la revisión de forma más justa

- Los artículos que pasan por un sistema de doble ciego mejoran siempre su calidad frente a otros modelos de arbitraje

\section{Aspectos negativos del método doble ciego}

- Es fácil identificar el autor o al menos la institución

- Elaborar una versión anonimizada complica la redacción del manuescrito y finalmente no ayuda a mejorar la calidad del artículo

- Revisiones normales y de doble ciego no presentaban diferencias en cuanto a las recomendaciones

- Ocultar la identidad no evita el sesgo ni varía la evaluación de los revisores ni la forma de recibir los comentarios de los autores

6. Críticas al modelo de doble ciego

Ahora, nos gustaría preguntarle por la alternativa que suponen los modelos de evaluación abierta. Para ello le preguntamos su grado de acuerdo con las siguientes afirmaciones: 
7. La posibilidad de ver el nombre de los autores/evaluadores aporta transparencia al proceso de evaluación

- Completamente de acuerdo

- De acuerdo

- Ni en acuerdo ni en desacuerdo

- En desacuerdo

- Completamente en desacuerdo

\section{EVALUACIÓN DE LA REVISTA ENCRUCIJADAS}

Ahora nos gustaría preguntar específicamente por el modelo de evaluación abierta que ha utilizado Encrucijadas en sus primeras seis ediciones. Nos gustaría conocer en qué medida cree usted que ha influido la evaluación en la redacción final de los artículos publicados en la revista atendiendo a su experiencia como autor o evaluador.

8. (SOLO PARA AUTORES) ¿En qué medida modificó su texto en función de los comentarios tras la evaluación por pares?

- Realicé cambios sustanciales en el artículo

- Realicé cambios menores en el artículo

- No realicé ningún cambio

9. (SOLO PARA EVALUADORES) Usted como evaluador, ¿cree que su revisión y recomendaciones se vio modificada al ser abierto el proceso?

- No

- Sí. En este caso, ¿en qué sentido?)

- El autor revisó convenientemente el texto en la línea indicada por los comentarios

- El autor revisó ligeramente el texto, aunque no siguió todas las indicaciones señaladas

- El autor hizo caso omiso de las recomendaciones

- No revise el estado final del texto, no puedo valorar las modificaciones realizadas

10. (AUTORES Y EVALUADORES) ¿En qué sentido crees que influyeron los comentarios?

11. Además de dejar visibles los nombres para autores y evaluadores, el proceso de evaluación ganaría calidad dejando visibles los comentarios de evaluación una vez termina el proceso de arbitraje.

- Completamente de acuerdo

- De acuerdo

- Ni en acuerdo ni en desacuerdo

- En desacuerdo

- Completamente de acuerdo 
12. En caso de que usted haya manifestado acuerdo con la pregunta anterior, ¿Qué tipo de limitaciones se deberían incluir para el acceso a la versión beta del artículo con los comentarios de sus evaluadores?

- Eliminar la posibilidad de ser detectado en motores de búsqueda (google, etc...)

- Otra:

13. ¿Considera que las mejoras al proceso de evaluación abierta podrían hacerse en otro sentido?

- Sí

- No

14. ¿Tiene alguna sugerencia de mejora?

15. ¿Qué opinión te merece la evaluación abierta respecto al método del doble ciego?

¿Tiene ventajas respecto al doble ciego?

¿E inconvenientes?

16. ¿Cómo valora en general proceso de evaluación abierta en Encrucijadas?

- Muy positivo

- Positivo

- Me es indiferente el modelo de evaluación

- Negativo

- Completamente negativo

17. ¿Tiene alguna sugerencia para el futuro de la evaluación en Encrucijadas? 